
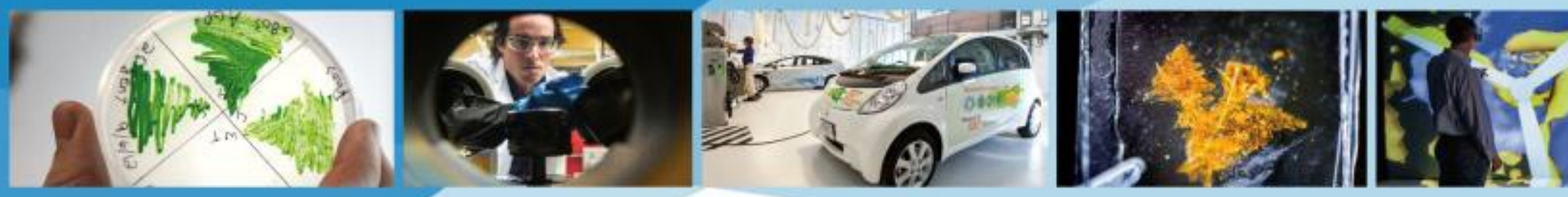

\title{
Modeling and Compensation Design for a Power Hardware-in-the-Loop Simulation of an AC Distribution System
}

Nathan Ainsworth, Ali Hariri, Kumaraguru Prabakar, Annabelle Pratt, Murali Baggu

Presented at the Fourth International Workshop on Grid Simulator Testing of Wind Turbine Drivetrains Golden, Colorado

April 25, 2017

N. Ainsworth, A. Hariri, K. Prabakar, A. Pratt and M. Baggu, "Modeling and compensation design for a power hardware-in-the-loop simulation of an AC distribution system," 2016 North American Power Symposium (NAPS), Denver, CO, 2016, pp. 1-6.

NREL/PR-5D00-68778

NREL is a national laboratory of the U.S. Department of Energy, Office of Energy Efficiency and Renewable Energy, operated by the Alliance for Sustainable Energy, LLC. 


\section{Introduction to Hardware-in-the-Loop Simulation}

- Hardware-in-the-loop (HIL) testing is performed for testing and benchmarking novel controllers and power hardware.

- HIL is performed in the following industries

- Automotive

- Aeronautics

- Power electronics

- Power systems.

- Different HIL

- Controller-hardware-in-the-loop (CHIL)

- Power-hardware-in-the-loop (PHIL)

- Combined.
Controller or hardware model development in software

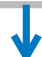

Testing developed models in software

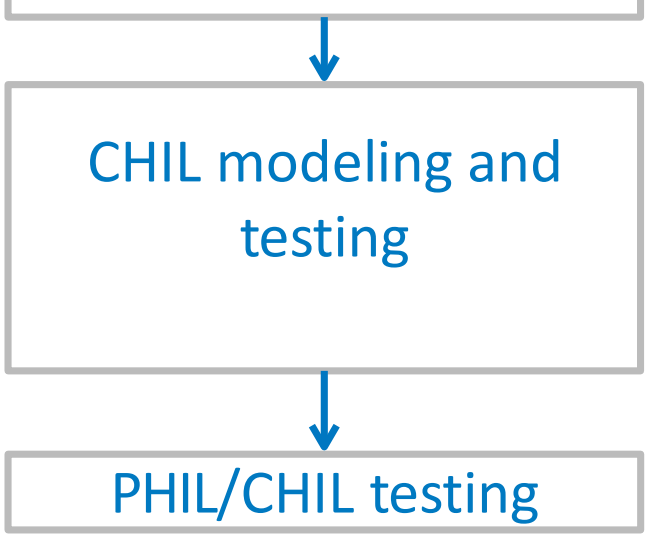

Typical controller/hardware development flow 


\section{Simplified CHIL \& PHIL Setup}

- CHIL: Simple

- PHIL: Complex and can become unstable

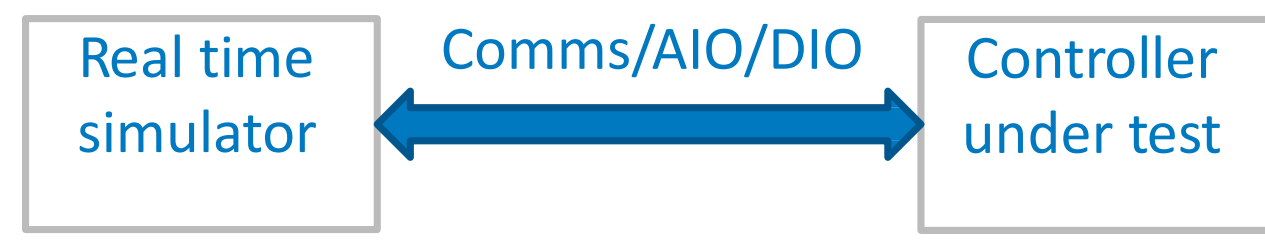

Controller hardware in loop

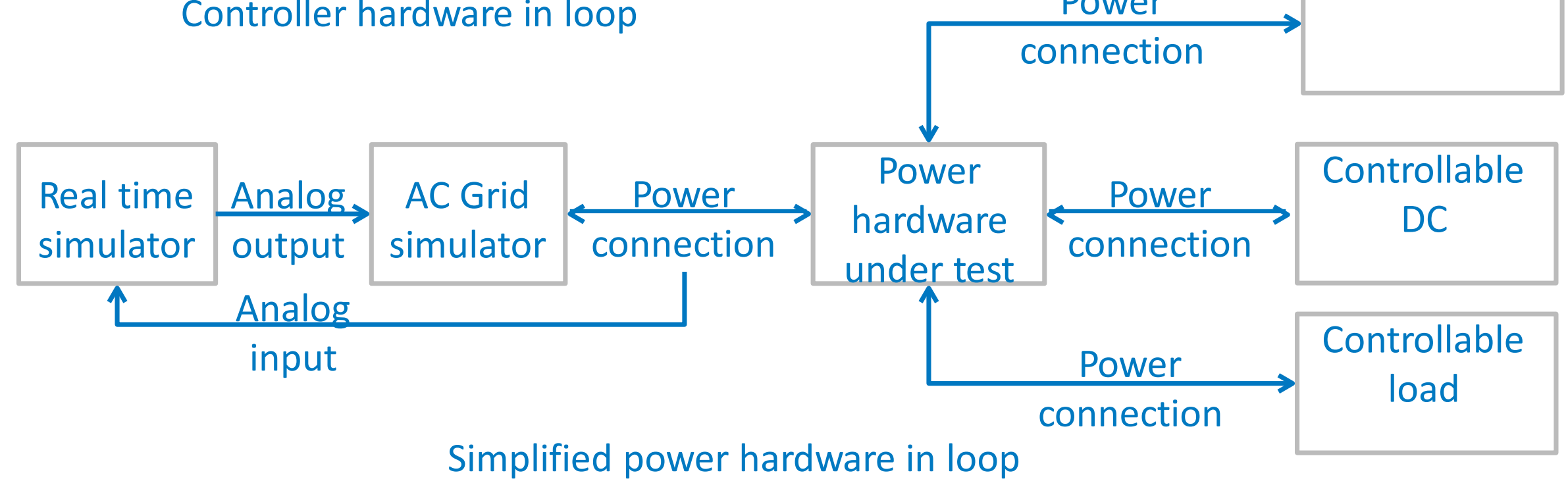




\section{Time Delay and Error Introduced in PHIL Testing}

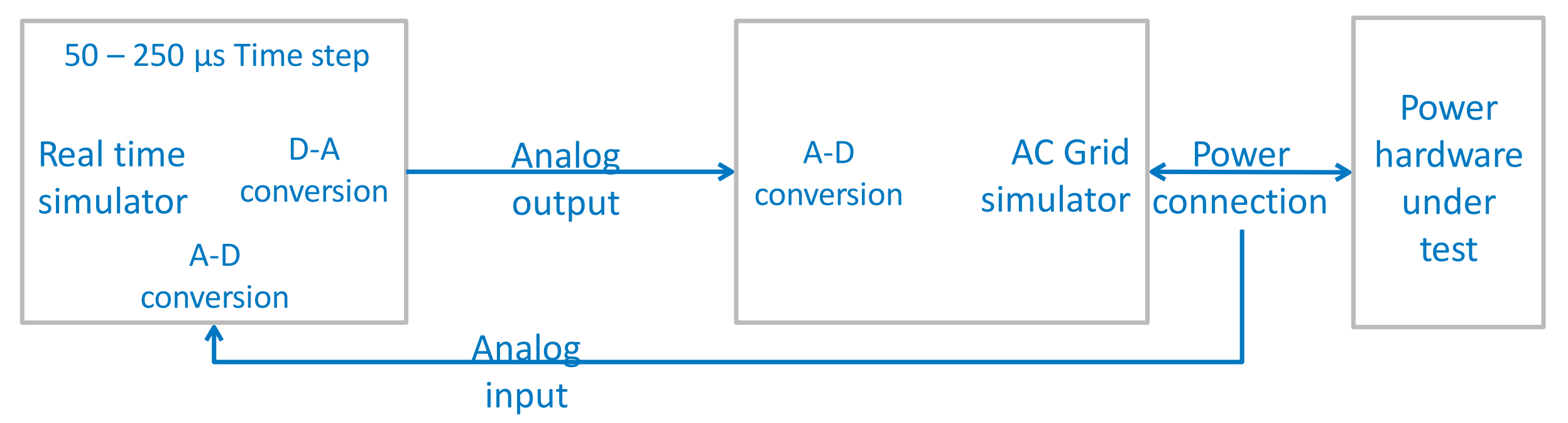

- Real time simulator runs at a time step of $50-250 \mu$ second time step.

- Delays are introduced by analog to digital and digital to analog conversion.

- Noise is present in the signal carrier adding more error in the testing. 


\section{Modeling of Distribution System in PHIL}

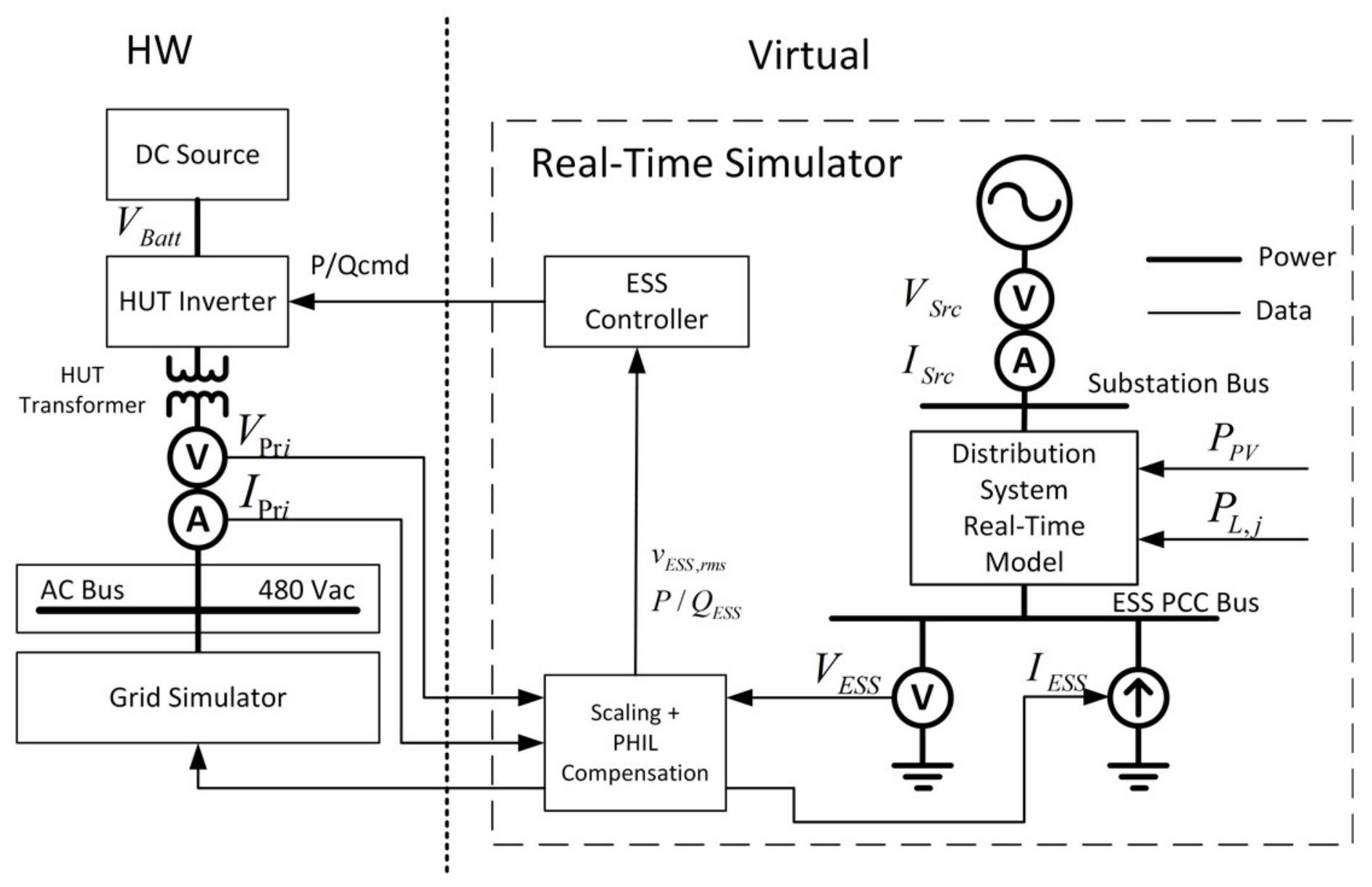

- HUT operates at $480 \mathrm{~V}$ 3- $Ф$, with a power rating of $450 \mathrm{kVA}$,

- Distribution feeder PCC is $12 \mathrm{kV}$ at 3-MVA rating. 


\section{Dynamic Modeling of PHIL Voltage and Current Loops}

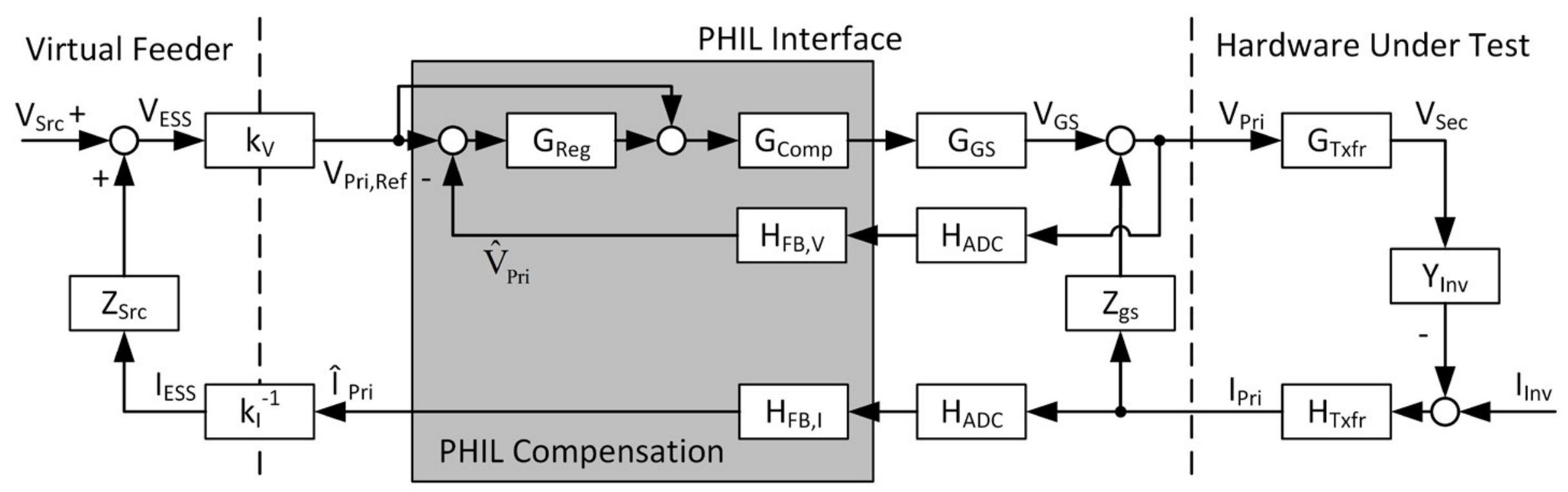

PHIL voltage and current loop control model

- Virtual feeder corresponds to the modeled distribution network.

- PHIL interface models the interface between RTS and power hardware. The delays and the proposed compensation are modeled here.

- Hardware under test models the hardware setup. 


\section{Modeling of Major Loop Components}

- Distribution feeder model

$$
V_{E S S}=V_{S r c}+Z_{S r c} I_{E S S}
$$

- Digital to analogue and analogue-to-digital converter

- Assumed to have unity gain. The delay introduced is modeled

$$
G_{D A C}=1 \quad H_{A D C}=e^{-s T_{R T S}}
$$

- Grid simulator - delay and a single pole filter

$$
G_{G S}=\frac{V_{\text {out }}}{V_{\text {ref }}}=\frac{1}{\frac{s}{2 \pi f_{G S}}+1} e^{-s T_{G S}}
$$

- Hardware under test transformer

$$
G_{T x f r}=H_{T x f r}=\sqrt{3} N_{T x f r}
$$

- Hardware under test inverter

$$
Y_{\text {Inv }}=\frac{2}{3} s C_{I n v} .
$$




\section{Uncompensated Stability and Performance}

- Open loop transfer function of conventional VITM

$G_{O L, V I T M}=\frac{k_{V} k_{I}^{-1} Z_{S r c}}{Z_{L}} e^{-s T_{G S}}$

- Uncompensated ESS PHIL loop transfer function
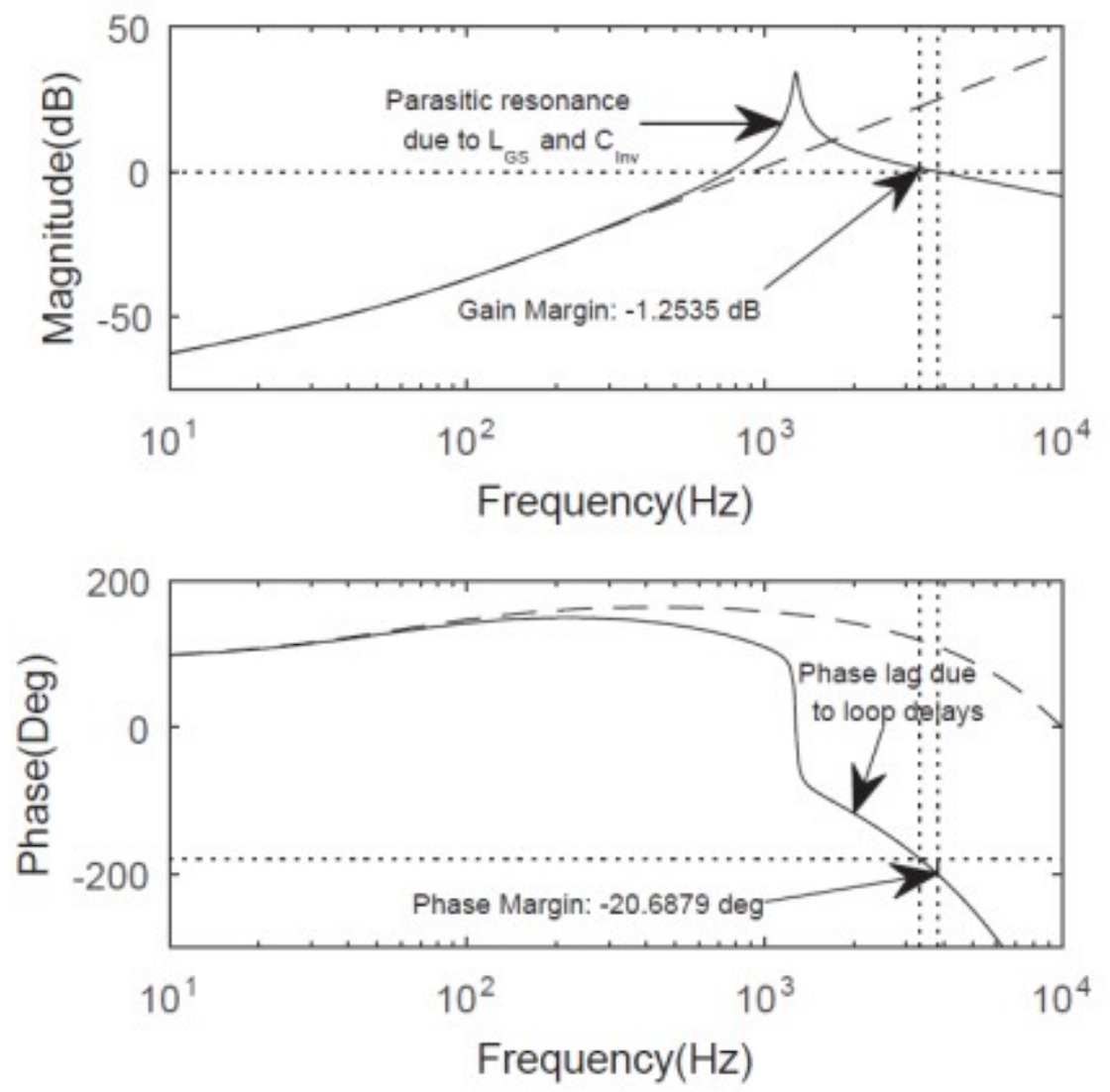

$G_{O L, U n c o m p}=\frac{k_{V} k_{I}^{-1} Z_{S r c}}{\left(\frac{s}{2 \pi f_{G S}}+1\right)\left(Z_{L}+Z_{G S}\right)} e^{-s\left(T_{R T S}+T_{G S}\right)}$

Bode plot of PHIL current loop without compensation (solid) versus conventional VITM model 


\section{Voltage Tracking Error}

- The uncompensated ESS PHIL have poor tracking at the AC fundamental frequency $(60 \mathrm{HZ})$

$$
\begin{aligned}
V_{\text {Error }}= & \frac{1+Z_{G S} H_{T x f r} Y_{I n v} G_{T x f r}-H_{A D C} G_{G S}}{1+Z_{G S} H_{T x f r} Y_{I n v} G_{T x f r}} V_{\text {Pri,Ref }} \\
& -\frac{H_{A D C} Z_{G S} H_{T x f r}}{1+Z_{G S} H_{T x f r} Y_{I n v} G_{T x f r}} I_{I n v}
\end{aligned}
$$

- This function exhibits $\sim 7 \%$ error under no load and $\sim 11 \%$ under loaded conditions

- It is desirable to compensate for the voltage error to ensure that $\mathrm{V}_{\text {pri }}$ accurately tracks $\mathrm{V}_{\text {Pri, Ref }}$ 


\section{Illusionary Real and Reactive Power Due to PHIL Setup}

- For the uncompensated PHIL loop,

$$
\tilde{S}_{E S S}=P_{E S S}+j Q_{E S S}=\tilde{V}_{E S S} \tilde{I}_{E S S}^{*}
$$

- Assuming unity magnitude for $\mathrm{G}_{\mathrm{Vff}}$ and $\mathrm{H}_{\mathrm{l}}$ at fundamental frequency

$$
\begin{aligned}
P_{E S S} & =k_{V}^{-1} k_{I}^{-1}\left[P_{P r i} \cos \delta-Q_{P r i} \sin \delta\right] \\
Q_{E S S} & =k_{V}^{-1} k_{I}^{-1}\left[Q_{P r i} \cos \delta+P_{P r i} \sin \delta\right]
\end{aligned}
$$

- Where $\delta=-\angle G_{V f f}\left(j \omega_{F}\right)-\angle H_{I}\left(j \omega_{F}\right)$

- Introduction of the phase shift $\delta$ by the PHIL loop at the fundamental frequency skews the value of $\mathrm{P}_{\mathrm{ESS}}$ and $\mathrm{Q}_{\mathrm{ESS}}$. This introduces illusionary $\mathrm{P}$ and $\mathrm{Q}$ of $8 \%$. 


\section{Design Requirements}

- The main issues that will affect the stability and accuracy of the PHIL simulation are:

- Instability of the PHIL current loop due to resonance and loop time delays

- Voltage difference between measured primary voltage $\left(V_{\text {Pri }}\right)$ and the commanded reference $\left(V_{\text {Pri,Ref }}\right)$ due to the parasitic impedance $Z_{G S}$

- Illusionary effect in $P$ and $Q$ due to the time delay

- Parasitic resonance due to the interaction between the grid simulator inductance $\mathrm{L}_{\mathrm{GS}}$ and the inverter filter capacitance $\mathrm{C}_{\text {inv }}$ 


\section{Proposed Compensation Method}

- Forward compensator $\left(\mathrm{G}_{\mathrm{comp}}\right)$ ensures stability of the current loop and addresses the resonance issue.

- Feedback filter $\mathrm{H}_{\mathrm{FB}, \mathrm{I}}$ and $\mathrm{H}_{\mathrm{FB}, \mathrm{V}}$ - accounts for the time delays in the interface and also eliminate the illusionary $\mathrm{P}$ and $\mathrm{Q}$ effect.

- Addition of the inner voltage regulation loop and voltage compensator $\mathrm{G}_{\mathrm{reg}}$, which will use a voltage feedback signal to enhance the voltage tracking capability 


\section{Dynamic Modeling of PHIL Voltage and Current Loops}

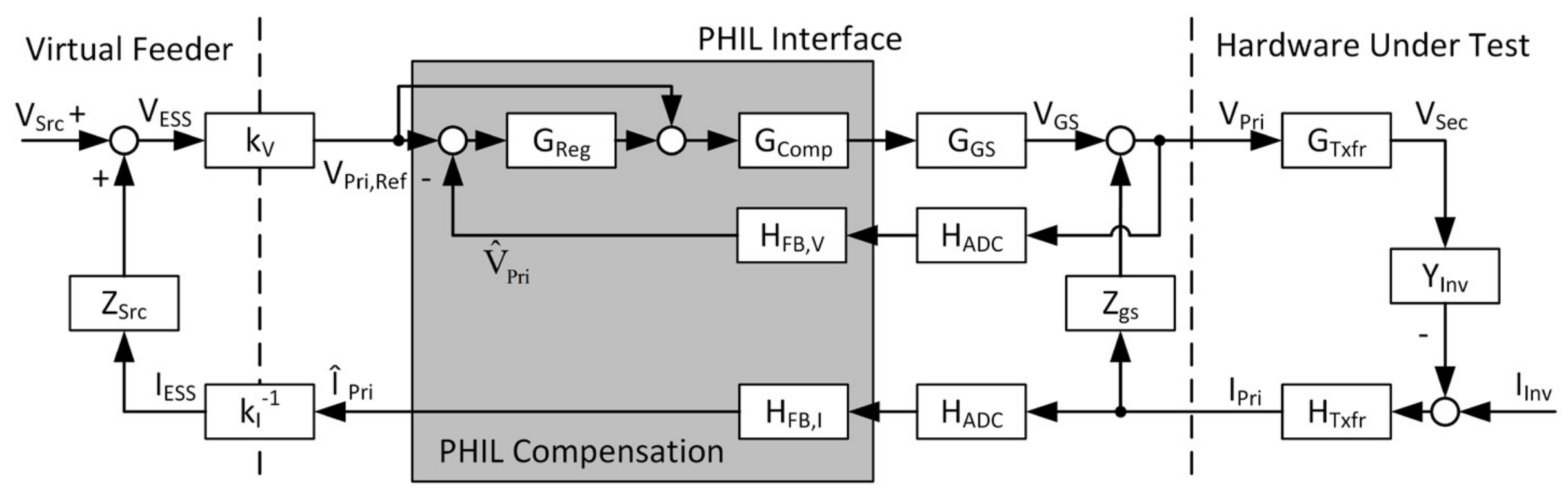

PHIL voltage and current loop control model

- Virtual feeder corresponds to the modeled distribution network.

- PHIL interface models the interface between RTS and power hardware. The delays and the proposed compensation are modeled here.

- Hardware under test models the hardware setup. 

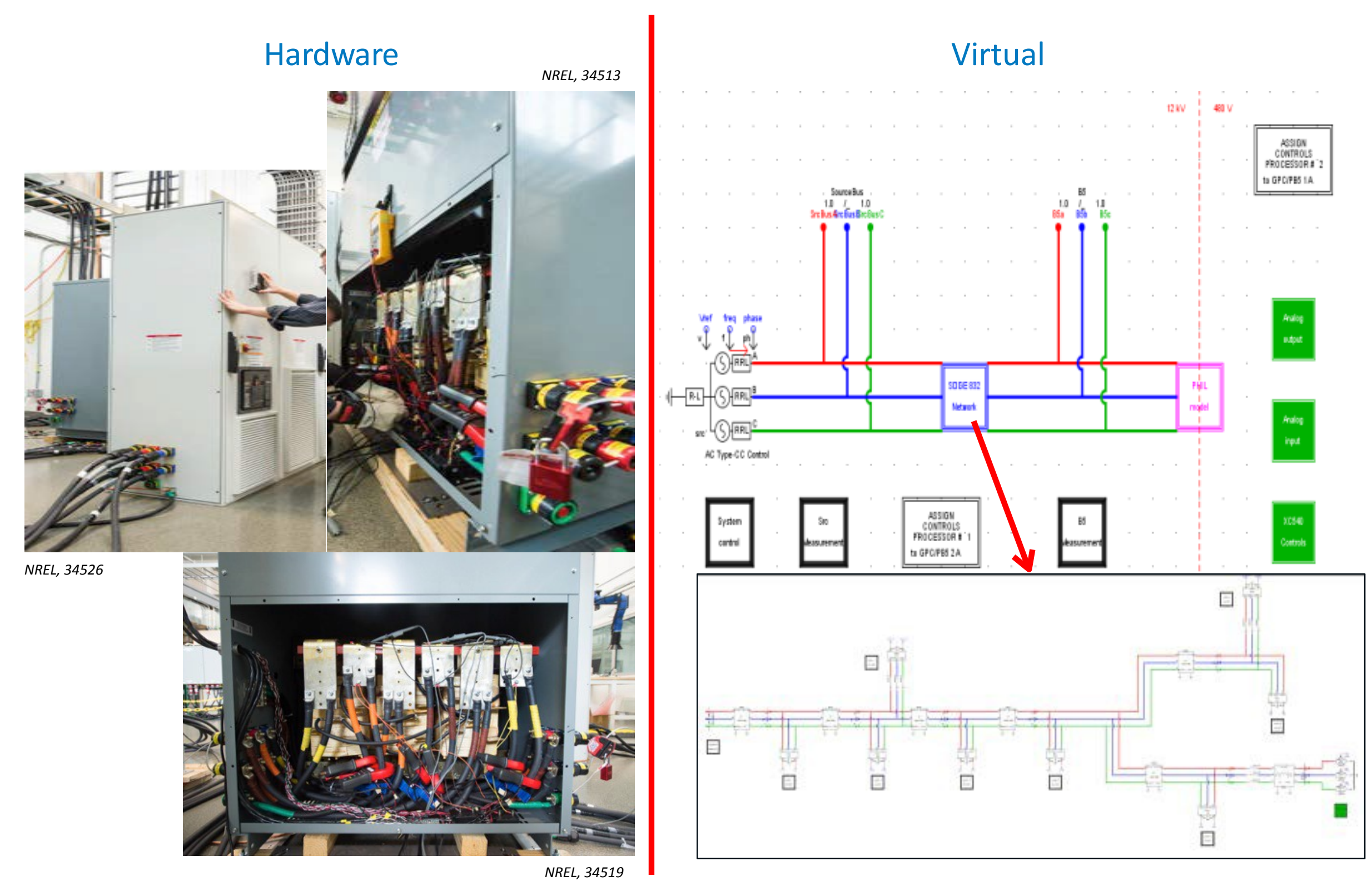


\section{SDG\&E CRADA: PHIL Stability and Accuracy Design}

\section{PHIL results without voltage regulation}

TABLE I: Test Results for Compensated PHIL Current Loop w/o Voltage Regulation

\begin{tabular}{|c|c|c|c|c|c|c|}
\hline P $_{\text {Inv }}$ & Q $_{\text {Inv }}$ & $\begin{array}{c}\mathrm{k}_{\text {V }} \mathrm{k}_{\mathbf{I}} \\
\mathbf{Q}_{\text {ESS }}\end{array}$ & $\mathbf{V}_{\text {Pri,Ref }}$ & $\mathbf{V}_{\text {Pri }}$ & $\mathbf{V}_{\text {Err }}$ & Q $_{\text {Err }}$ \\
\hline $\mathrm{kW}$ & $\mathrm{kVAr}$ & $\mathrm{kVAr}$ & Vrms & Vrms & $\%$ & $\%$ \\
\hline 99.6 & 9.3 & 22.2 & 274.9 & 278.0 & 17.5 & -13.4 \\
\hline 199.8 & 11.0 & 40.5 & 275.0 & 279.3 & 18.3 & -15.2 \\
\hline-102.8 & -8.5 & -16.2 & 274.7 & 275.6 & 18.8 & -22.4 \\
\hline-205.5 & -5.2 & -30.6 & 274.6 & 274.0 & 19.2 & -29.9 \\
\hline
\end{tabular}

$\mathrm{PHIL}$ results with voltage regulation

TABLE II: Test Results for Compensated PHIL Current Loop w/ Voltage Regulation

\begin{tabular}{|c|c|c|c|c|c|c|}
\hline P Inv $_{\text {Inv }}$ & $\begin{array}{c}\mathbf{k}_{\text {V }} \mathbf{k}_{\mathbf{I}} \\
\mathbf{Q}_{\text {ES }}\end{array}$ & V $_{\text {Pri }, \text { Ref }}$ & V Pri $_{\text {Pri }}$ & $\mathbf{V}_{\text {Err }}$ & Q $_{\text {Err }}$ \\
\hline $\mathrm{kW}$ & $\mathrm{kVAr}$ & $\mathrm{kVAr}$ & Vrms & Vrms & $\%$ & $\%$ \\
\hline 99.8 & 7.3 & 5.9 & 274.9 & 275.0 & 0.6 & 1.4 \\
\hline 200.0 & 2.2 & 9.0 & 275.0 & 275.0 & 0.6 & -3.4 \\
\hline-102.7 & -5.6 & -4.1 & 274.7 & 274.8 & 0.5 & -3.3 \\
\hline-205.5 & -5.0 & -9.0 & 274.7 & 274.8 & 0.6 & -7.8 \\
\hline
\end{tabular}

Voltage error reduced to $<1 \%$

Reactive power error reduce to $<7 \%$ 


\section{Closed-loop PHIL Voltage Control Filter}

Smoother waveforms

Uneven peak
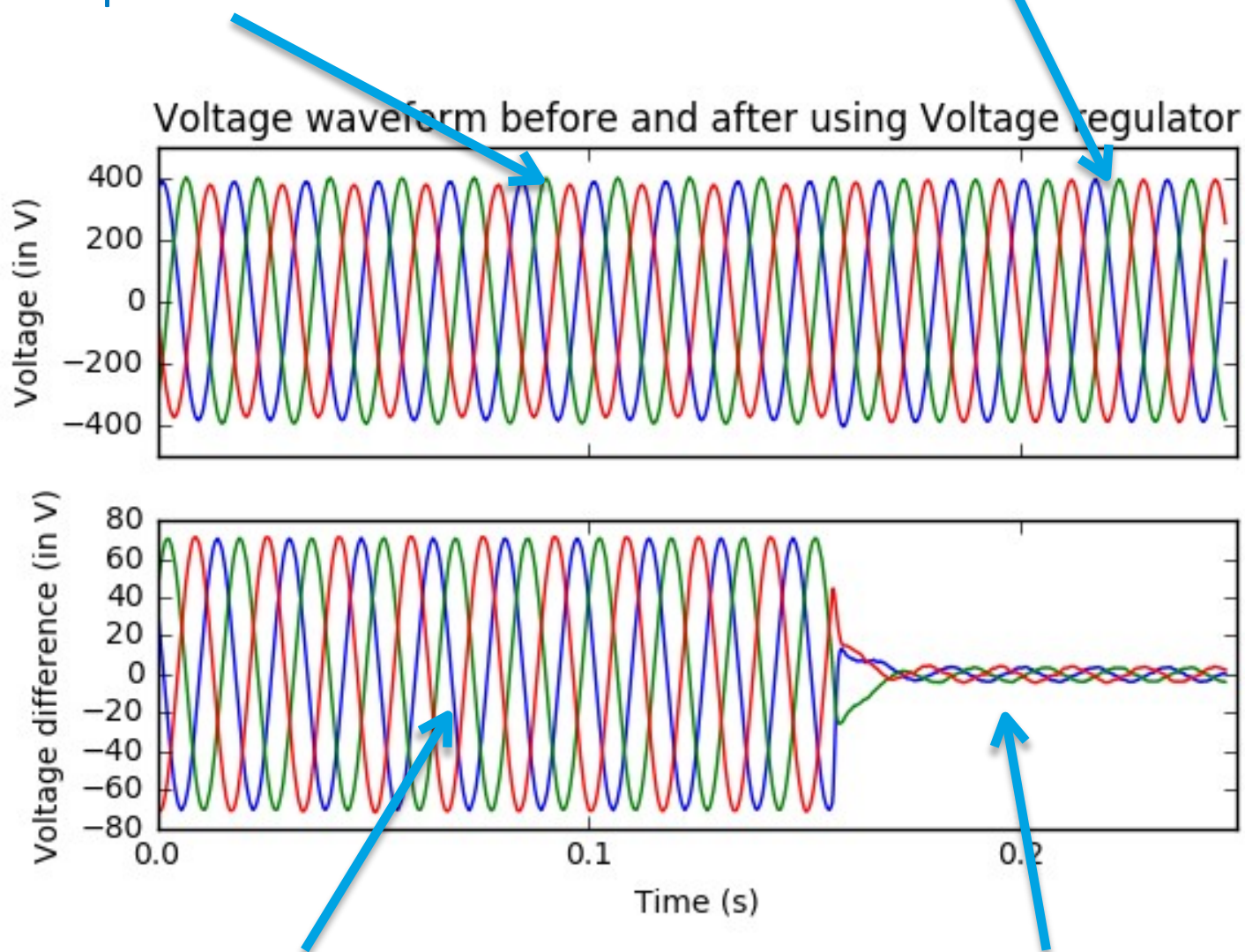

Difference between

After turning on voltage regulator

set point and observed voltage 


\section{Summary}

- Models for PHIL test setup was developed for hardware ESS inverter and simulated distribution system.

- Uncompensated PHIL loop exhibited instability under some operating conditions due to parasitic effects and loop delays.

- Compensation method based around a voltage regulation loop and forward and feedback compensators was presented.

- The proposed method was validated both analytically and experimentally to provide stable PHIL system with improved accuracy. 


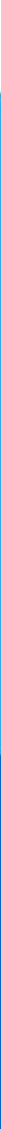

NREL is a national laboratory of the U.S. Department of Energy, Office of Energy Efficiency and Renewable Energy, operated by the Alliance for Sustainable Energy, LLC. 